\title{
Performance experiment and numerical prediction on the optimization design of the micro-sized water turbine for the hose reel irrigator
}

\author{
Lingdi Tang*, Shouqi Yuan, Yue Tang, Haofang Yan \\ (Research Center of Fluid Machinery Engineering and Technology, Jiangsu University, ZhenJiang 212013, China)
}

\begin{abstract}
In order to significantly improve the efficiency of driving water turbine used in hose reel irrigator, a new water turbine structure was proposed by the method of performance test and numerical calculation. The internal flow characteristics of original water turbine were analyzed, and it was found that unreasonable design of main flow passage components such as inlet, outlet and runner could not effectively translated pressure energy of upper stream into impact kinetic energy of blade, and gave rise to low energy conversion efficiency of water turbine. The inadequate internal flow and uneven pressure distribution were also not conducive to energy conversion efficiency. Then a new structure of water turbine structure was presented, in which the inlet has a tangential nozzle jet and the outlet is in axial direction. The computational analysis showed that the nozzle jet at the inlet of the new water turbine runner, which makes jet flow mainly concentrate in the impacted blade passage, can reduce the loss of flow kinetic energy. The axial outflow increases the distance of inflow in the runner, which is more conducive to the runner blades work. Performance experiments on both original and new water turbines showed that the highest efficiency of the new turbine is almost 20 percentages higher than that of the original turbine, and the new turbine is nearly triple output power over the original turbine. The internal flow characteristic analysis and the performance experiment were conducted to assess the feasibility of the replacement of the original water turbine by the new water turbine.
\end{abstract}

Keywords: micro-sized water turbine, hose reel irrigator, optimization design, experimental study, numerical analysis DOI: $10.25165 /$ j.ijabe.20181105.2876

Citation: Tang L D, Yuan S Q, Tang Y, Yan H F. Performance experiment and numerical prediction on the optimization design of the micro-sized water turbine for the hose reel irrigator. Int J Agric \& Biol Eng, 2018; 11(5): 108-115.

\section{Introduction}

Sprinkling irrigation is an effective water-saving irrigation method. The water evaporating in the sprinkling irrigation changes the climate of the farmland to restrain plant transpiration and soil evaporation effectively to improve the rate of the water-resource use $\mathrm{e}^{[1,2]}$. Sprinkling irrigation also has positive effect on improvement of salinized soil by salt leaching ${ }^{[3,4]}$. Sprinkling irrigation machinery owns the multiple structural types, including center pivot irrigator, lateral moving irrigator, hose reel irrigator, etc ${ }^{[5,6]}$. Thus, the hose reel irrigator has good mobility, high automation and high adaptability to sprinkle the different-sized fields.

The hose reel irrigator came into use in Europe in the 1960s and is still used today, the manufacturing enterprises concentrate mainly in Italy, Austria, France and Germany ${ }^{[7,8]}$. The traditional hose reel irrigator was mainly composed of sprinkler cart, water pipe, hose, driving device (water turbine), gear box, and so on.

Received date: 2016-09-27 Accepted date: 2018-04-22

Biographies: Shouqi Yuan, Professor, research interests: theory, design and computational fluid dynamics of pumps and fluid machinery, design of low-energy sprinkler unit, design and industrialization for the key device in the new type spray drip irrigation system, Email: shouqiy@ujs.edu.cn; Yue Tang, Professor, research interests: design, development and industrialization of the intelligence agricultural irrigation equipment, Email: tomt@ujs.edu.cn; Haofang Yan, Associate Professor, research interests: water saving irrigation theory and technology, agricultural water management, Email: yanhaofang@ yahoo.com.

*Corresponding author: Lingdi Tang, $\mathrm{PhD}$, research interests: engineering applications and performance improvement of agricultural irrigation equipment, fluid machinery design and engineering techniques. Research Centre of Fluid Machinery Engineering and Technology, Jiangsu University, No.301, Xuefu Road, Zhenjiang 212013, China. Tel: +86-15952898606, Email: lingdit@ ujs.edu.cn.
Among them, the water turbine was the key component driving the irrigator. However, in China, the issue of low efficiency of the water turbine for this irrigator is remarkable. Tang et al. ${ }^{[9]}$ and Yuan et al. ${ }^{[10,11]}$ built the performance test apparatus for water turbine to test the hydraulic performances of the turbines in the hose reel irrigator with the homegrown types of JP50 and JP75. The experimental results showed that the peak efficiency of JP50 type was less than $20 \%$, and that of JP75 type was less than $10 \%$. Then the CFD method was used to analyze the inner flow structure in water turbine. The analysis indicated that the improper designs of the major past components in the turbine including input channel, output channel, and turbine impeller, obstructed the inner water flow and caused the uneven pressure distribution in the turbine. This improper design was the main cause of the low efficiency of the water turbine.

The water turbine used in hose reel irrigator is a simple micro-sized water turbine with the low specific speed of no more than $20 \mathrm{~m} \cdot \mathrm{kW}$ working in pressure system with higher backpressure, which has an analogous working condition with the mixed flow turbine and the pump as turbine (PAT). Chen et al. ${ }^{[12]}$ investigated the working characteristics of the water turbine used in driving cooling tower fan with the specific speed ranges at 40-60 $\mathrm{m} \cdot \mathrm{kW}$. They proposed that the mixed flow turbine could be chosen appropriately in the pressure system. The working fluid in the impulse turbine cannot discharge smoothly from the flow channel, and the vortex was formed in the blade passage. This vortex consumed water energy and induced vibration to reduce the efficiency of the turbine. In practice, most of water turbines working in the pressure system employ the structure of the mixed flow turbine. Zhang et al. ${ }^{[13,14]}$ studied the hydraulic characteristics of the mixed flow water turbine used in driving cooling tower fan with the specific speed of $50 \mathrm{~m} \cdot \mathrm{kW}$. They 
revealed that hydraulic loss mainly generated in diversion components, especially in the stand ring, next was in the runner part. The predicted efficiencies under all the six operating condition exceeded $73 \%$. Zheng et al. ${ }^{[15]}$ designed a Francis turbine with the specific speed of $53 \mathrm{~m} \cdot \mathrm{kW}$. They adopted the blade torsion resistance of $18^{\circ}$ with uniform thickness, the nose angle of $345^{\circ}$ with rectangular spiral case, and the cone angle for draft tube of $13^{\circ}$. The predicted efficiency of this turbine was $83 \%$. Li et al. ${ }^{[16]}$ also designed a Francis turbine with the specific speed of $53 \mathrm{~m} \cdot \mathrm{kW}$ by using the binary theory. The predicted efficiency of the turbine was more than $80 \%$. The PAT is also widely employed in the pressure system. Jain and Patel ${ }^{[17]}$ gave the graphic of applicable scope for the different kinds of the PATs, and illustrated that the research on the low flow rate in the PAT with low specific speed was rare. Derakhshan et al. ${ }^{[18]}$ derived the relation of between the pump and the PAT, based on the velocity triangle in theory. Derakhshan et al. ${ }^{[19]}$ also employed the four centrifugal pumps with the specific speed ranges from 14 to 60 to test the performance characteristics of both the pump and the PAT. They compared the characteristic curves of the four pumps and illustrated that the head ratio and the flow rate ratio of the pump and the PAT varied in the pumps with different specific speeds. Shinhama et al. ${ }^{[20]}$ improved the PAT efficiency by designing the impeller with thinner and round shaped vane inlet and clarified that the head-flow rate curve varied little with the impeller outlet diameter reducing. González et al. ${ }^{[21]}$ and Fernández et al. ${ }^{[22]}$ investigated numerically the characteristics of the inner flow fields in a double-suction centrifugal pump and a single stage centrifugal pump respectively, when both pumps were working in the turbine mode. Singh et al. ${ }^{[23,24]}$ studied experimentally some methods to improve the PAT efficiency by changing the entry angle of the impeller and expanding the impeller export and the draft tube. Derakhshan et al. ${ }^{[25,26]}$ designed optimally the blade profile of a PAT by genetic algorithm, neural network and the gradient-based optimization algorithm.

The above literatures revealed that the impulse turbine working in the pressure system produced the flooded pressure jet flow to cause the high kinetic energy loss instead of the free jet flow. The disc friction loss in the pressure system is higher than that in the non-pressure system, so the turbine efficiency in pressure system is lower. The mixed flow turbine is often used in the pressure system with modest backpressure, but there is rare research on the turbine with the specific speed less than $40 \mathrm{~m} \cdot \mathrm{kW}$. The PAT with the ultra-low specific speed has the narrow high efficiency area, its efficiency drastically decreases when the flow rate deviates from the high efficiency area.
In order to improve and exceed the hydraulic performance of original water turbine, this paper analyzed its internal flow characteristic using CFD (computational fluid dynamics) technique, found and identified the key factors and parts that caused its low efficiency. Then a new structure of water turbine structure was presented aiming at unreasonable inlet and runner type, in which the inlet has a tangential nozzle jet and the outlet is in axial direction. The inlet pressure was reduced in the new water turbine under the condition of certain load power and outlet pressure. The analysis of internal flow characteristic and comparison experiment for new water turbine were conducted to understand the energy conversion mechanism of efficiency improvement, then to achieve the purpose of replacing the original water turbine.

\section{Materials and methods}

\subsection{Experimental apparatus}

A series of experiments were designed and conducted to study the output shaft powers and the work efficiencies of the original JP75 water turbine and the new structural water turbine under the various working water heads and runner rotational speeds. These experiments were also used to compare the performance characteristics of the two water turbines and to validate the accuracy of the numerical methodology adopted in the paper. The testing apparatus was composed of a water conveying pump, a tested water turbine, a magnetic powder brake, a torque-revolution speed transducer, a pressure sensor, an electromagnetic flowmeter, pipeline and the data acquisition system. The schematic representation of the whole system is shown in Figure 1. The working water was pumped from the reservoir by a centrifugal pump with the rated flow of $50 \mathrm{~m}^{3} / \mathrm{h}$ and the head of $80 \mathrm{~m}$. Two pressure gauges in mechanical spring type were mounted at the inlet and the outlet of the water turbine to measure the pressure drop. The inlet valve of the water turbine was used to regulate the inlet flow rate, and the outlet valve of the water turbine was used to control the outlet pressure. The combination of inlet and outlet valves could keep the working water head in test value. The experimental data, including the runner rotating speed, the shaft torque and the water-turbine flow rate, can be collected under the different turbine loads regulated by the magnetic powder brake. Then the output shaft power and the work efficiency of the tested water turbine can be calculated. The pressure gauge, the electromagnetic flowmeter and the torque-revolution speed transducer used in the experiments have estimated experimental uncertainties of $0.25 \%, 0.5 \%$ and $0.5 \%$ respectively, and the magnetic powder brake has a maximum load of $20 \mathrm{~N} \cdot \mathrm{m}$.

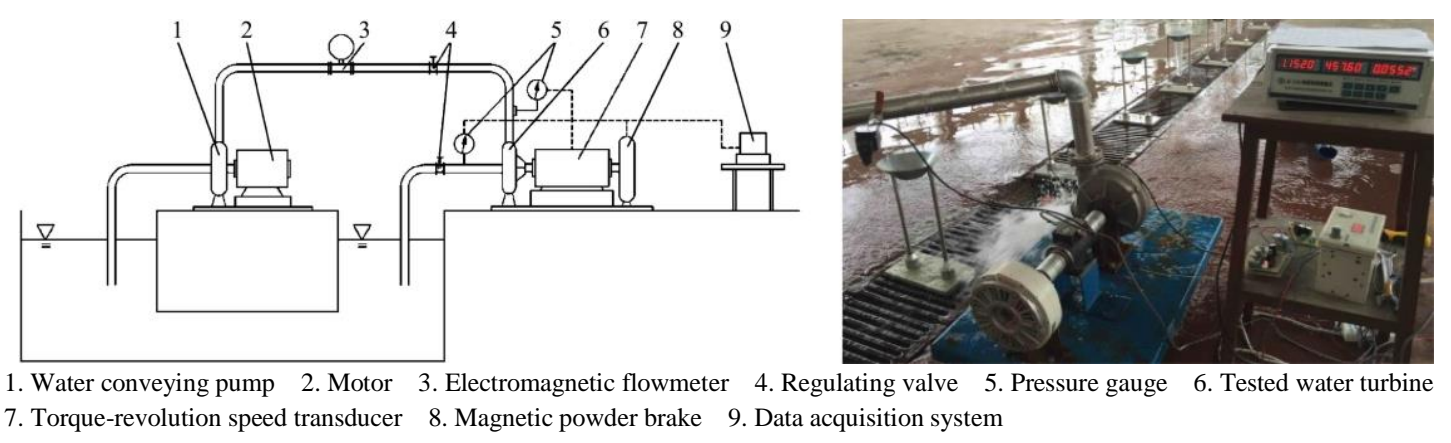

Figure 1 Schematic diagram of experimental apparatus

\subsection{Numerical strategy}

The numerical calculation method was adopted to study the energy utilization ratios of flow passage components in the original
JP75 water turbine to design the new water turbine structure with the high energy utilization ratio. The geometrical parameters of the original and new water turbine structures considered in this 
study are listed in Table 1. The two structural schemes were built by the CATIA software, as showed in Figure 2. The grids for the two water turbines were generated by the meshing tool ICEM CFD with the blocking method, and the numbers of grid elements were 5043624 and 3963978 for the original structure and the new one respectively in Figure 3. The independence of grid number was proven by comparing the simulation results with different numbers of grid elements. As shown in Figure 4, the prediction of the shaft output power experiences slight change when the grid number reaches about $3.96 \times 10^{6}$.

Table 1 Specifications of runners

\begin{tabular}{lcc}
\hline Design Parameters & original & new \\
\hline Outer Diameter $D_{1} / \mathrm{mm}$ & 276 & 190 \\
Inner Diameter $D_{2} / \mathrm{mm}$ & 168 & 70 \\
Runner Height $b / \mathrm{mm}$ & 29 & 28 \\
Blade Thickness $\delta / \mathrm{mm}$ & 4 & 5 \\
Number of Blades $Z$ & 24 & 12 \\
\hline
\end{tabular}
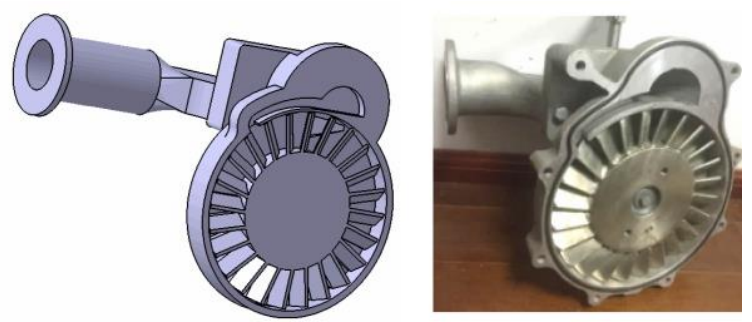

a. Water turbine type 1
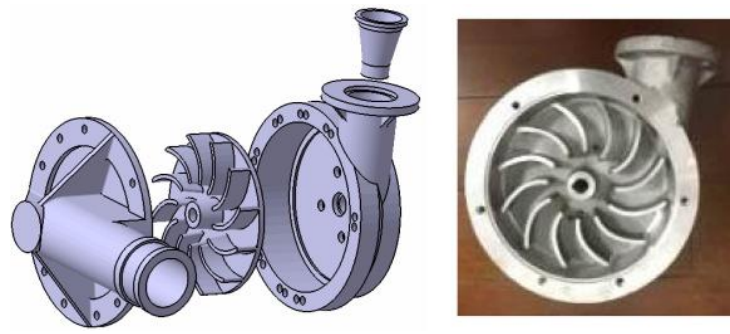

b. Water turbine type 2

Figure 2 Geometrical configurations of two types water turbines

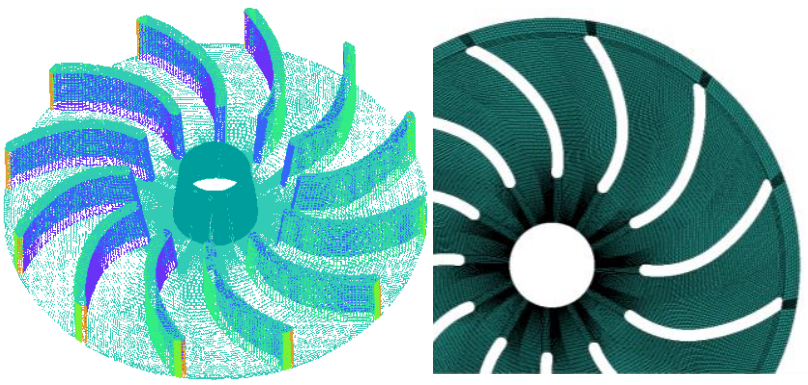

Figure 3 Part of computational grid

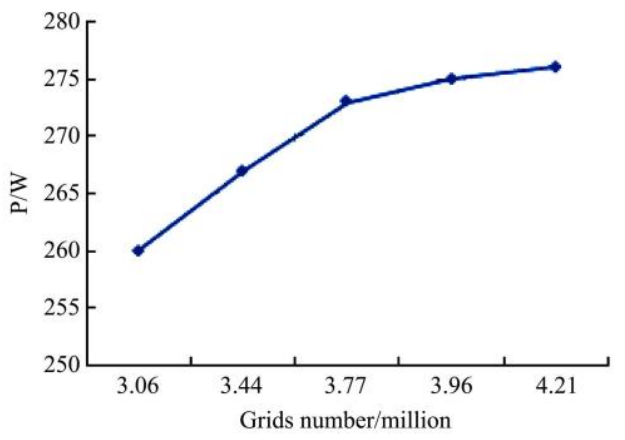

Figure 4 Grid independence analysis
Three-dimensional steady and unsteady flow analyses were performed by the general software ANSYS CFX to study the distribution of the flow field and the water energy utilization for the two water turbines. The discretization in space used second order scheme and in time chosen second order backward Euler scheme. The runner was rotational component while the other parts were considered under stationary. All the parts were attached by the domain interface using the frozen rotor mixing model in steady analyses and the transient rotor stator mixing model in unsteady analyses. The inlet boundary condition was set to normal speed at the inlet tube corresponding to the turbine operation flow rate. The outlet condition was defined by an opening at the draft tube outlet with a relative static pressure of $0.1 \mathrm{MPa}$. And non-slip boundary condition was applied to all the walls with smooth wall condition. The convergence criteria for all variables were set as $10^{-5}$. The time step was considered as $6^{\circ}$ of revolution and the duration time was the length of the runner rotating eight revolutions for the unsteady flow simulation. The Shear Stress Transport (SST) $k$ - $\omega$ turbulence model was employed on account of the curved passage and the rotational flow.

\subsection{Experimental validation}

Experimental data for the output shaft power of the water turbine were collected to verify the accuracy of the numerical calculation. Figure 5 shows that the numerical calculation results have an acceptable agreement with experimental data. The mean deviation between the calculation results and experimental values of the output shaft power is $5.2 \%$. Therefore, the numerical calculation method used in this study is correctly implemented, and the other calculations can be conducted in the prediction of the water-turbine flow field.

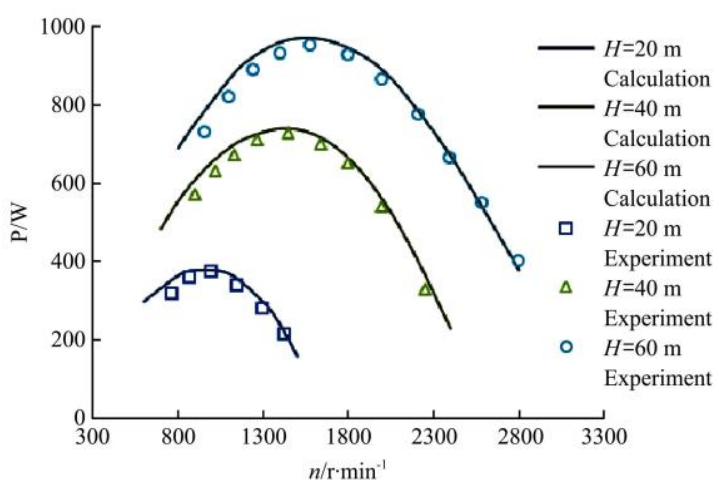

Figure 5 Comparison of experimental results and model predictions

\section{Results and discussion}

\subsection{Original water turbine}

The performance characteristics of the original JP75 water turbine were tested by the experiment, as shown in Figure 6. The output shaft power of this turbine ranges from $20 \mathrm{~W}$ to $250 \mathrm{~W}$ with the working water head increasing from $13 \mathrm{~m}$ to $30 \mathrm{~m}$, while the rotational speed of the turbine shaft increases from $100 \mathrm{r} / \mathrm{min}$ to $600 \mathrm{r} / \mathrm{min}$. The noteworthy problem in the original turbine is the low turbine efficiency (the maximum efficiency is $11 \%$ ).

The water energy consumption in the original water turbine was studied to analyze the turbine efficiency. The water turbine used in the hose reel irrigator has four flow parts converting the working water energy, including inlet tube, jet, runner, and draft tube as shown in Figure 7. The runner is the main component using water energy to convert energy to the shaft power (58.49\%) 
and does useful work. The jet has the big energy conversion due to the flow impact loss. The inlet tube and the draft tube use less water energy to overcome the flow friction in the flow

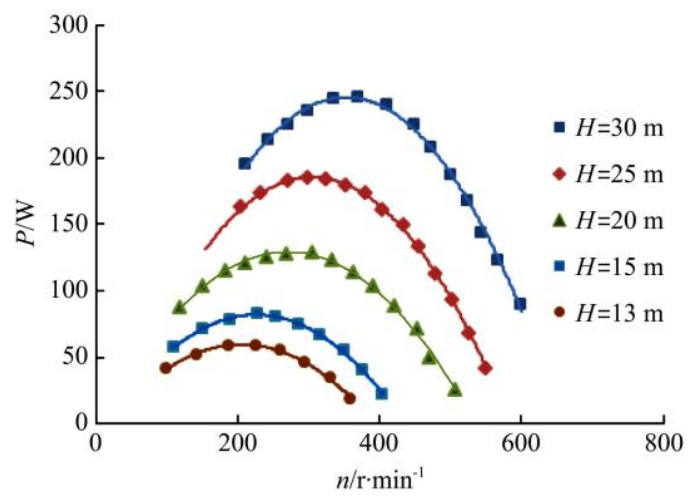

a. Power -rotational speed characteristic channel. Obviously the runner is the most important flow part in the turbine and is optimally designed to increase the turbine efficiency.

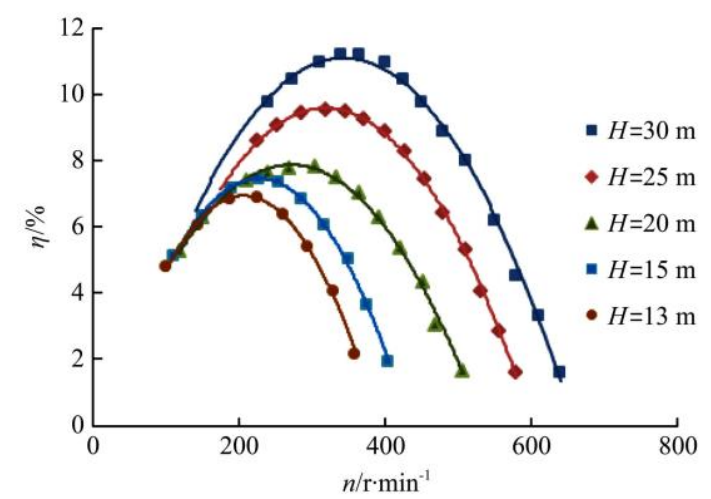

b. Efficiency-rotational speed characteristic

Figure 6 Speed characteristic curves of the original water turbine

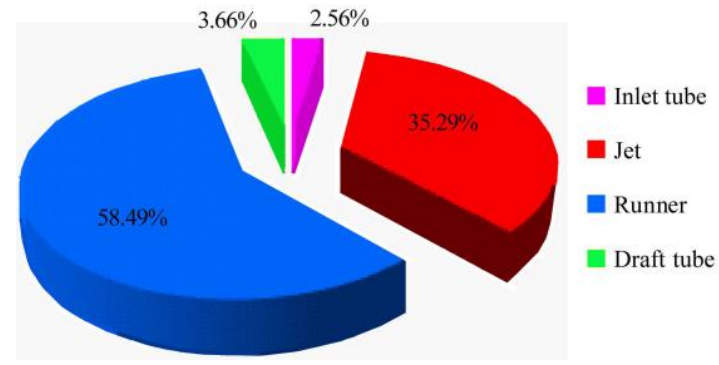

Figure 7 Energy distribution in the original water turbine

The flow characteristics in the turbine runner were numerically calculated to study the effect of the runner structure. The working water from the jet outlet impacts on the runner to drive it, and then flows out from the main outlet and the bypass outlet, as showed in Figure 8 . The pressure contour for the middle section of the runner in Figure 8 shows the uneven distribution in the radial surface of the runner. To analyze the pressure distribution inner the runner, a dimensionless pressure coefficient $C_{\mathrm{p}}$ is defined as follows:
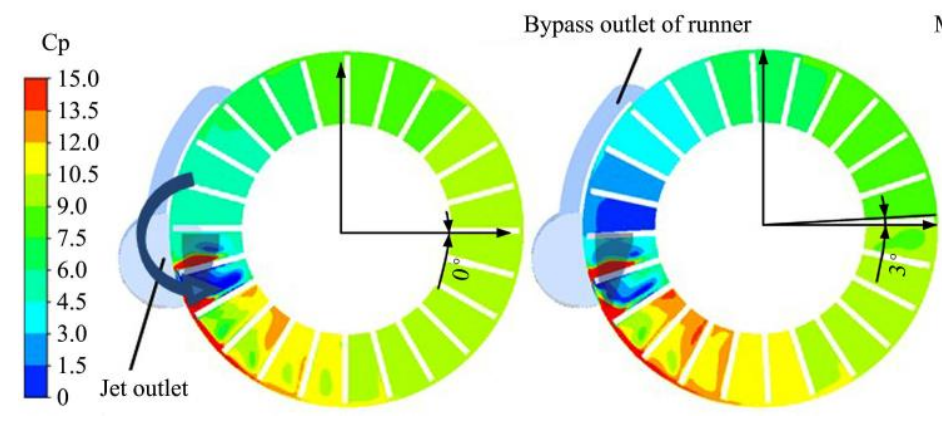

$$
C_{\mathrm{p}}=\frac{P-P_{\mathrm{ref}}}{0.5 \rho v^{2}}
$$

where, $P_{\text {ref }}$ is the minimum pressure, $\mathrm{Pa} ; \rho$ is the mass density of work flow, $\mathrm{kg} / \mathrm{m}^{3} ; v$ is the velocity, $\mathrm{m} / \mathrm{s}$.

The pressure inner the runner decreases along the runner rotation direction. The jet flow rushing into the runner inlet causes the high pressure in fractions of the two facing passages, and then some low pressure regions appear after the high pressure flow impact. The jet exit covers two flow passages, and neither locates at the minimum cross section of the compressed tube nor focuses on a single flow passage. The main outlet of the runner locates opposite to the runner inlet (the jet outlet), while the bypass outlet of the runner locates about four passages away from the main one. This structure disorders the steady pressure distribution and makes the low pressure area cover several passages from the runner inlet to the bypass outlet. Hence, the maximum and minimum pressure regions occur in the same flow passage to cause the unstable flow. The pressure variation induces the varying force in the diametral plane to result in the additional energy loss.
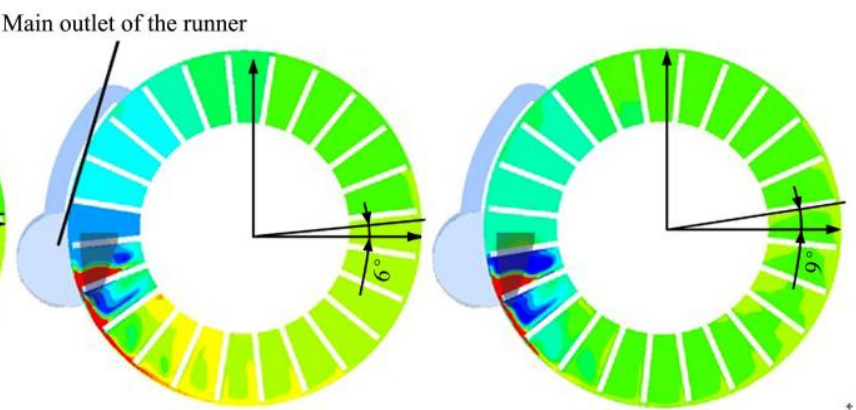

Figure 8 Pressure distribution at mid-span of runner for the original water turbine

\subsection{New water turbine}

The new water-turbine structure was proposed, and its inlet tube, jet, runner, and draft tube were optimally designed to improve the turbine performance characteristics. Figure 9 shows the tested performance characteristics of the new water turbine. The output shaft power of this turbine ranges from $80 \mathrm{~W}$ to $1000 \mathrm{~W}$ with the working water head increasing from $15 \mathrm{~m}$ to $60 \mathrm{~m}$, while the rotational speed of the turbine shaft increases from $700 \mathrm{r} / \mathrm{min}$ to $2600 \mathrm{r} / \mathrm{min}$. The highest turbine efficiency is about $40 \%$, while the lowest efficiency is $12.5 \%$ and is greater than the highest efficiency of the original water turbine.
The water energy consumption in the new water turbine was studied in Figure 10. The energy conversion in the runner represents $68.1 \%$ contributing to improve the turbine efficiency and enhances the energy efficiency (10\%). The new water turbine combines the jet like that in turbo turbine and the runner like that in mixed-flow turbine, as shown in Figure 1b. The jet outlet position was changed to the radial direction of the runner, and the jet outlet was designed to be the minimum cross section after shrinking as the jet. The position of the runner outlet was changed to the runner center to make the work water go through more blades and do more useful work. 


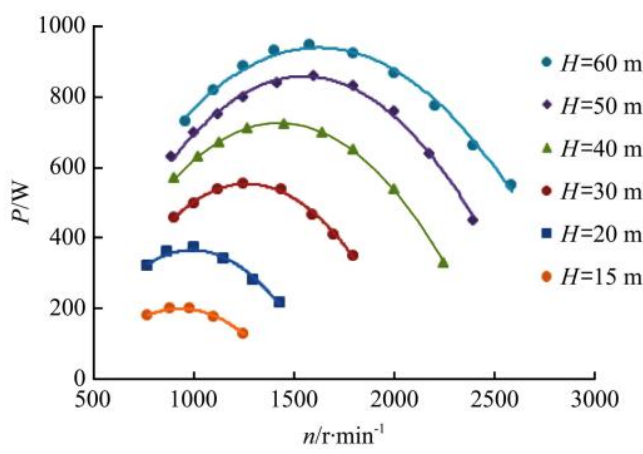

a. Power -rotational speed characteristic

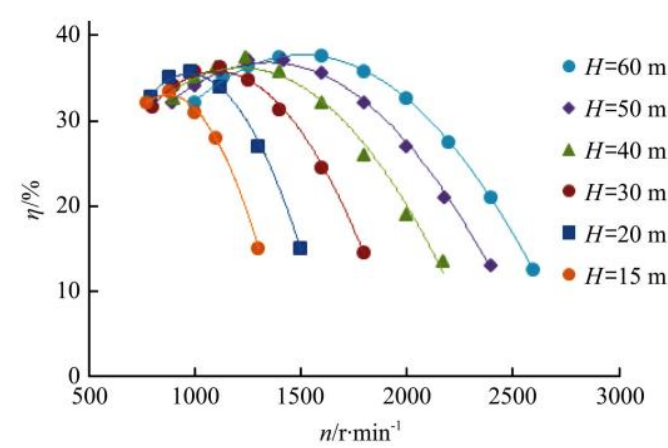

b. Efficiency -rotational speed characteristic

Figure 9 Speed characteristic curves of the new water turbine

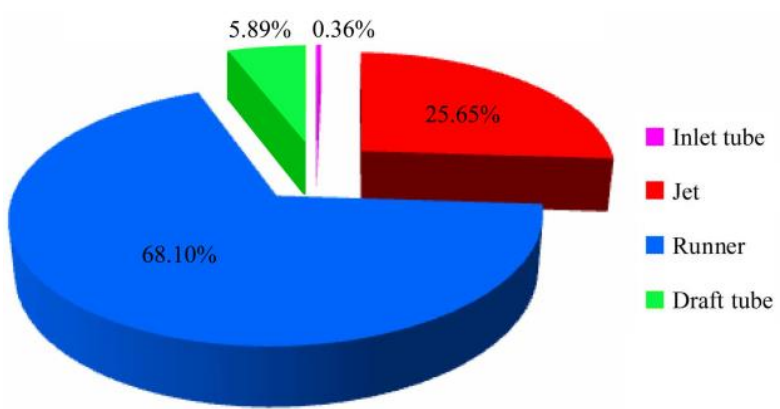

Figure 10 Energy distribution in the new water turbine

The pressure distributions at mid-span of the runner during rotating one-twelfth revolution are shown in Figure 11. The pressure changed smoothly in the runner domain expect for the areas around the jet flow. The high-pressure jet flow went into the blade passage, and then the pressure gradually fell off from the runner inlet to the runner outlet due to the conversion water energy from the jet flow to the runner mechanical energy. The pressures near the downstream tip clearance and the flow passage before the jet flow were slightly higher than the zone away from the jet flow. The low pressure zone appeared in the flow passage after the flow impulse passage, especially on the blade back and the tip clearance.
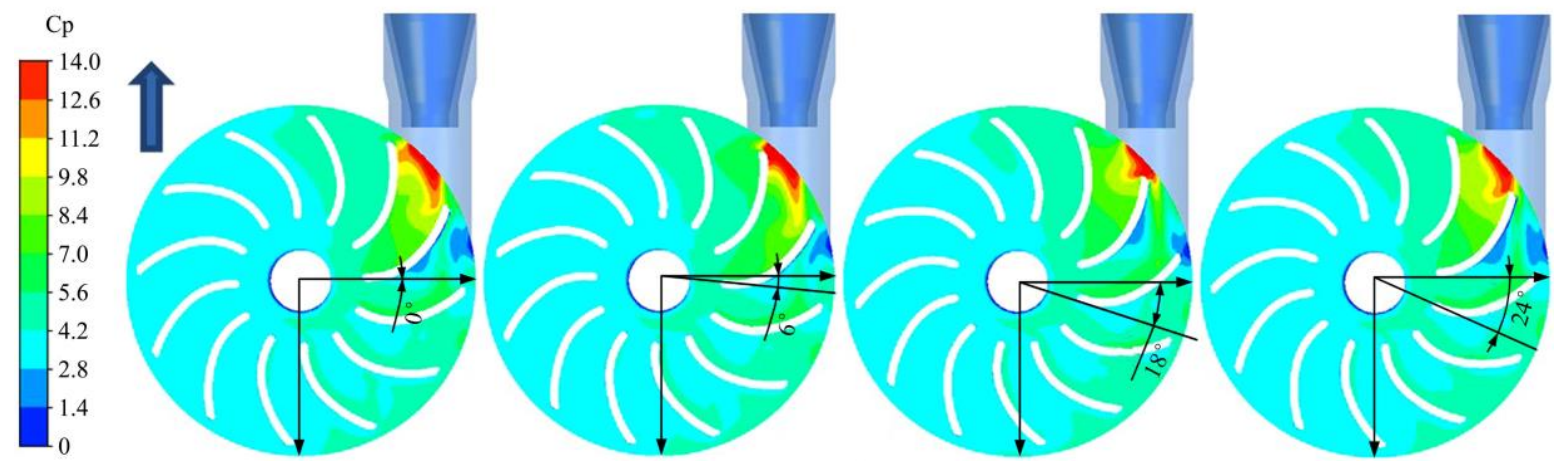

Figure 11 Pressure distribution at mid-span of the new runner

The turbine performance characteristics are determined by the impulsed flow in the blade passage. Five monitoring points were selected in the blade passage to study the impulse flow behavior and the water energy conversion. They were arranged at the runner base circles with the equal interval, as shown in Figure 12. The P1 was located at the blade tip with the circle diameter of $190 \mathrm{~mm}$, while the P5 was located at the blade root with the circle diameter of $70 \mathrm{~mm}$ in Table 2.

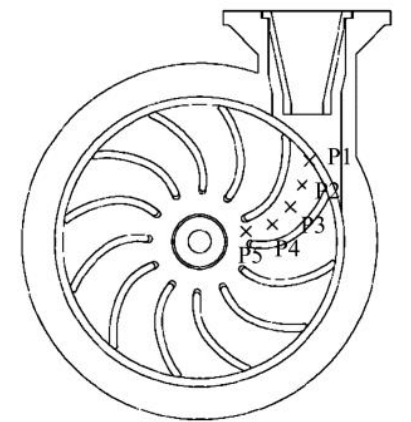

Figure 12 Monitoring points located in the blade passage

Table 2 Monitoring point locations

\begin{tabular}{cccccc}
\hline Monitoring point & P1 & P2 & P3 & P4 & P5 \\
\hline Circle diameter/mm & 190 & 160 & 130 & 100 & 70 \\
\hline
\end{tabular}

Figure 13 presents the pressure variations at five monitoring points with the runner rotating. The maximum pressure values are presented in the blade passage hit by the jet flow, and the pressure magnitude drops with the decrease of the circle diameter. Then the pressure values in the circles gradually reduce to the same level with the runner rotating. The pressure drop in the circle represents the water energy conversion. Figure 14 shows pressure drops at the five monitoring points with the runner rotating. The pressure drop gradually reduces with the circle diameter decreasing. The maximum pressure drop and the minimum one are presented at the $\mathrm{P} 1$ and the $\mathrm{P} 5$ respectively. The drop at the P1 is almost three times that at the P5. The impact flows in the large-diameter circles do more useful work to contribute to increase the turbine shaft power.

The above discussed pressure is the total pressure in the turbine, and the total pressure is consisted of the static pressure and the velocity. The total pressure in the turbine represents the gross water energy, while the static pressure and the velocity represent the static energy and the kinetic energy respectively. Figure 15 presents the values of the total pressure, the static pressure and the velocity at the five monitoring points. The velocity coefficient $K_{v}$ is defined to study the velocity field:

$$
K_{\mathrm{v}}=v / \sqrt{2 \times g \times H}
$$


where, $v$ is the velocity, $\mathrm{m} / \mathrm{s} ; g$ is the gravitational acceleration, $\mathrm{m} / \mathrm{s}^{2} ; H$ is the total head, $\mathrm{m}$.

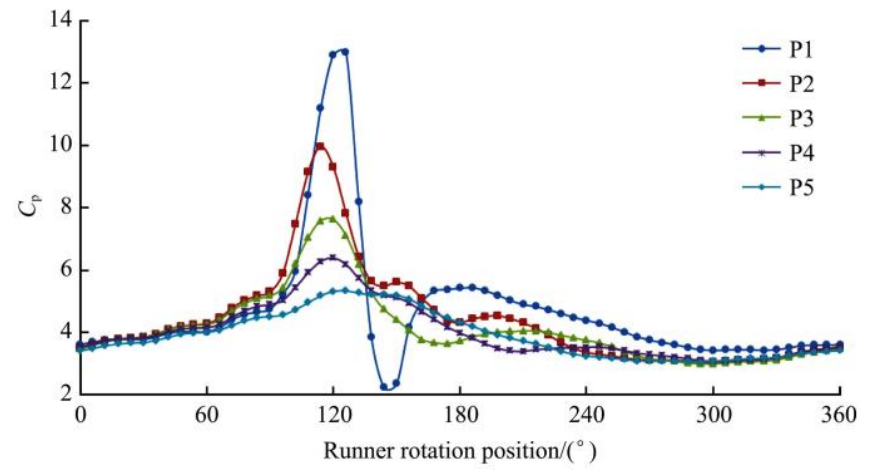

Figure 13 Pressure variations at the monitoring points

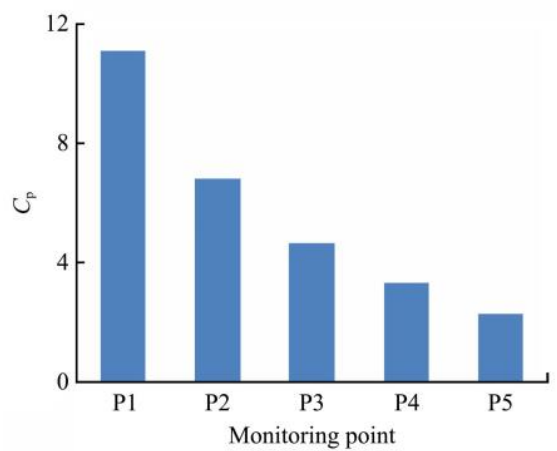

Figure 14 Pressure drops at monitoring points

The rapid decrease in the total pressure and the velocity are appeared at the locations from the tip to the middle of the blade. The gross energy and the kinetic energy have the similar distribution along the blade passage, while the static energy keeps stability essentially. The water energy converting to the runner mechanical energy is determined by the kinetic energy.

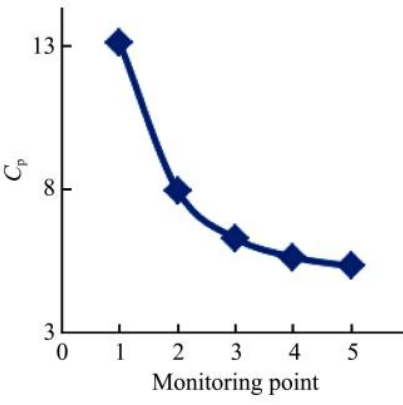

a. Total pressure

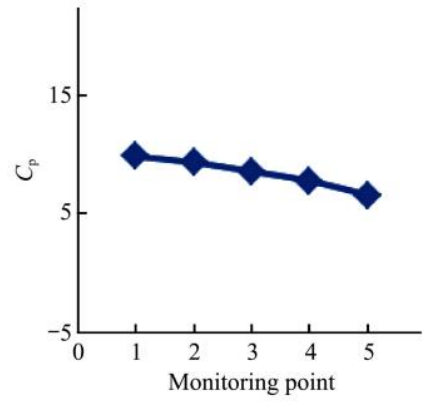

b. Static pressure

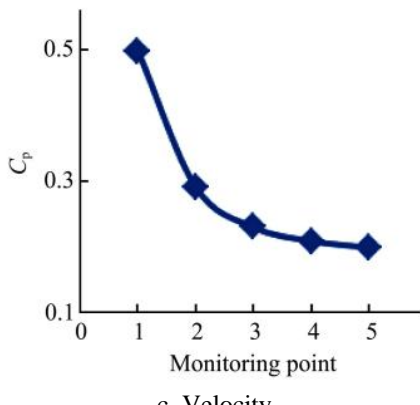

c. Velocity

Figure 15 Physical characteristics in the flow field

\subsection{Comparison of the two structural water turbines}

The flow characteristics in the runner inlet as the main work area, was studied for the two water turbines in Figures 16 and 17. The impact flows in the blade passage are presented obviously by the streamlines in Figure 16. The large vortex flow is appeared in the impacted passage of the original turbine, while the new turbine has the steady flow in the impacted passage. Some water from the jet outlet flows along the vertical to the passage in the original turbine, while most of the flow in the new turbine impact on the blade along the passage to do more work. Figure 17 shows the velocity vectors in the impacted flow fields of the two water turbines. The water from the jet of the original turbine flows in all directions, while the jet flow on the mainstream concentrates on the impacted blade passage in the new turbine. The water energy utilization rate of the new turbine is higher than that of the original turbine from the turbine structural perspective.

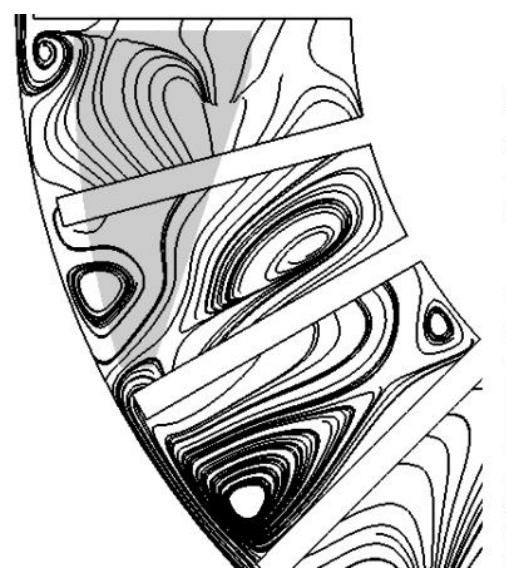

a. Original

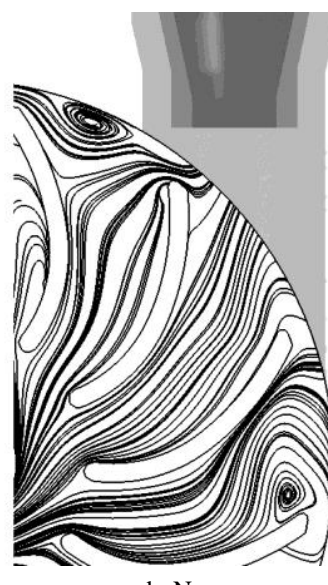

b. New
Figure 16 Streamline at mid-span of the runner inlet
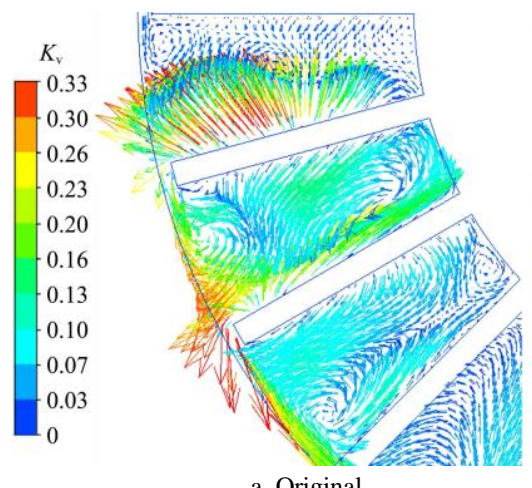

a. Original

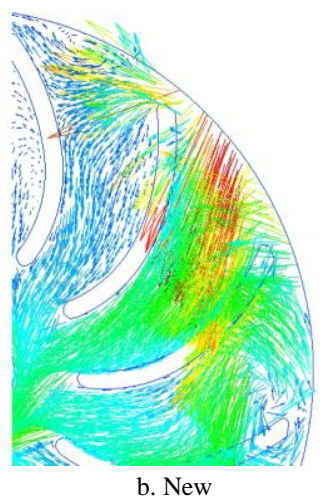

b. New
Figure 17 Velocity vector distribution at mid-span of the runner inlet

Figure 18 presents the pressure variations at the blade tip points with the runner rotating for the two water turbines. The pressure evolution has the similar tendency for the two water turbines, but the pressure in the original turbine is characterized by obvious pulsation. This pulsatile change in the pressure shows the unsteady flow in the original turbine, and the new structural turbine was designed to improve the stability of the energy conversion.

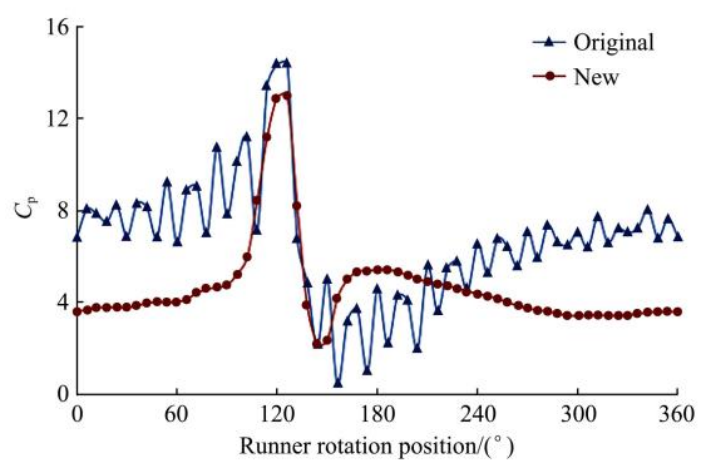

Figure 18 Pressure variations at the blade tip points 
The force situation of the runner is one of the key factors for the operation reliability of the water turbine. The radial force coefficient $K$ was defined to study the radial force on the runner as follows $^{[27]}$ :

$$
K=\frac{F}{0.5 \pi \rho D b u^{2}}
$$

where, $F$ is the radial force, $\mathrm{N} ; D$ is the runner diameter, $\mathrm{m} ; b$ is the width of runner inlet, $\mathrm{m} ; u$ is the circumferential velocity in the largest base circle of runner, $\mathrm{m} / \mathrm{s}$.

The radial force distributions during one revolution for the two structural runners are depicted in Figure 19. The radial force on the original runner has periodical change, while the radial force on the new runner has approximately the same size with the runner rotating. The radial force on the new runner is smaller than that on the original runner. The maximum radial force value of the original runner is 1.5 times that of the new runner, while the minimum radial force value of the original runner is 1.25 times that of the new runner, as shown in Figure 20.

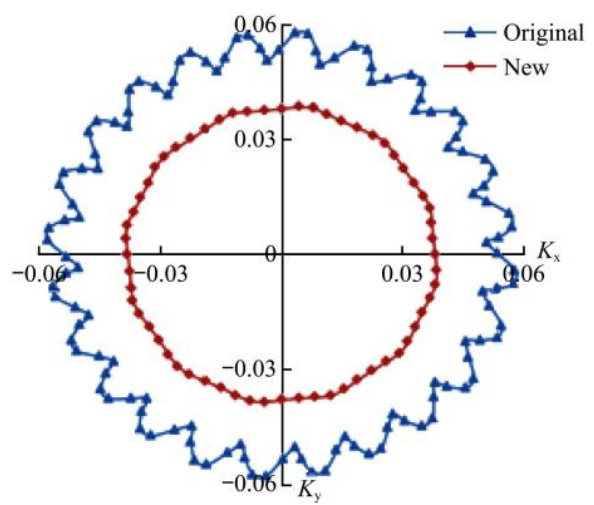

Figure 19 Radial force on the runner

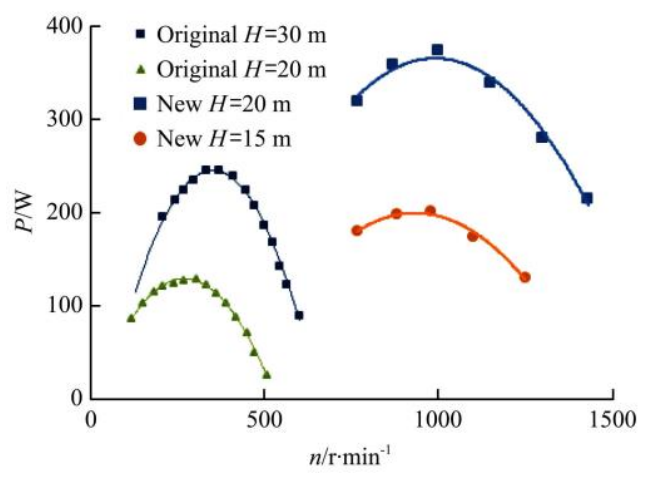

Figure 21 Speed characteristic curves of the two water turbines
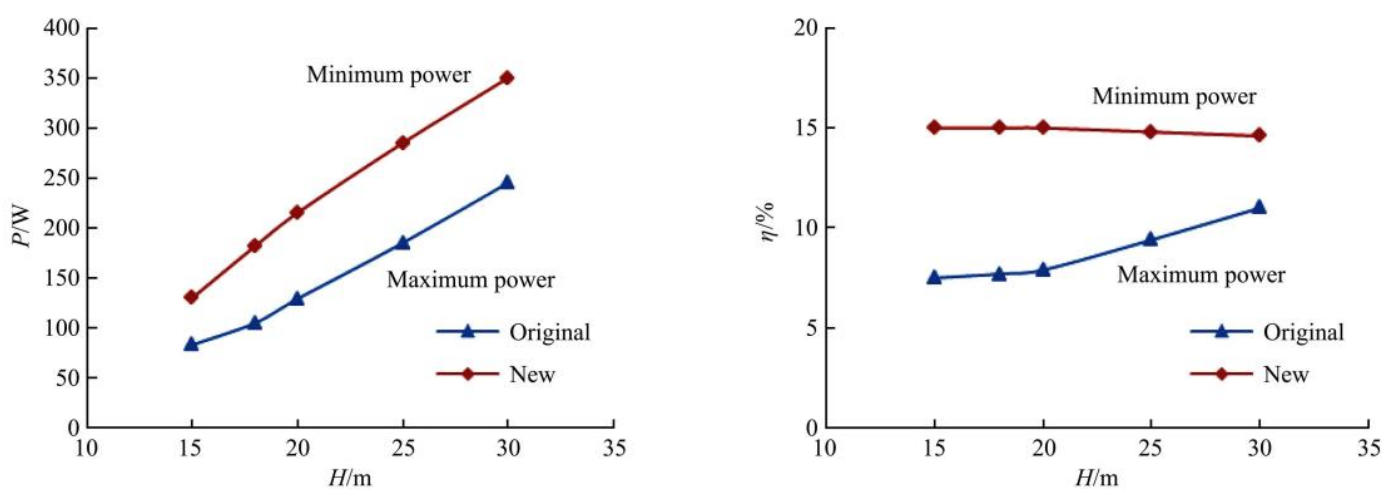

Figure 22 Comparisons in performance characteristics under the same head

\section{Conclusions}

The performance characteristics and the flow characteristics of

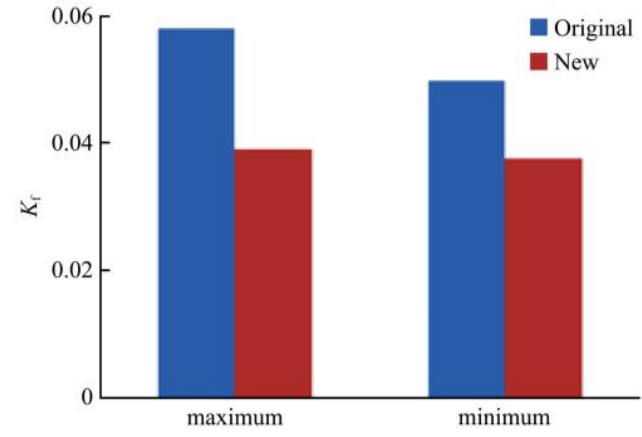

Figure 20 The values of radial forces

The performance characteristics represent the output performance of the water turbine. Figure 21 presents the tested performance characteristics of the two turbines under the partial working water heads including some high heads of the original turbine and some low heads of the new turbine. The output rotational speed of the original turbine is lower than $600 \mathrm{r} / \mathrm{min}$, while the rotational speed of the new turbine is higher than $700 \mathrm{r} / \mathrm{min}$. The output shaft power and the turbine efficiency of the new turbine are also higher than that of the original turbine. Especially, the minimum output power and the minimum efficiency of the new turbine are higher than the maximum power and the maximum efficiency of the original turbine under the same working water head, as shown in Figure 22. With the head increasing from $15 \mathrm{~m}$ to $30 \mathrm{~m}$, the minimum power of the new turbine is almost $100 \mathrm{~W}$ higher than the maximum power of the original turbine, while the minimum efficiency of the new turbine is almost $8 \%$ higher than the maximum efficiency of the original turbine. Therefore the new turbine structure was advanced to improve the turbine output performance and the turbine efficiency significantly.

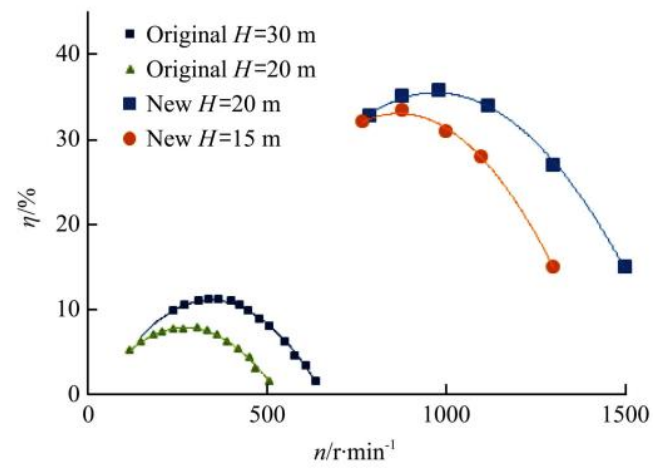


water energy to the shaft power, due to the analysis for the energy conversion in the turbine. The unreasonable design for the runner inlet and outlet is a major cause of the low efficiency.

A new structural water turbine was proposed with maximizing the water energy utilization in the runner. The jet outlet was designed along the radial direction of the runner, and the runner outlet was designed in the runner center to lengthen the work blade. The water energy efficiency of the runner is improved by approximately $10 \%$. The maximum turbine efficiency can reach $40 \%$ due to the tested performance characteristics.

Comparing the flow characteristics in the two structural water turbines, the jet flow on the mainstream concentrates on the impacted blade passage in the new turbine, while the water from the jet outlet of the original turbine flows into the runner in all directions. The pressure at the monitoring point of the original turbine is characterized by obvious pulsation, while the steady pressure variation is monitored in the new turbine. Based on comparison of the performance characteristics of the two turbines, the minimum efficiency of the new turbine is almost $8 \%$ higher than the maximum efficiency of the original turbine under the same water head.

\section{Acknowledgements}

This work was supported by the National Key Research and Development Program (2016YFC0400202).

\section{[References]}

[1] Zhao W X, Li J S, Li Y F. Modeling sprinkler efficiency with consideration of microclimate modification effects. Agricultural and Forest Meteorology, 2012; 161: 116-122.

[2] Cavero J, Medina E T, Puig M, Martínezcob A. Sprinkler irrigation changes maize canopy microclimate and crop water status, transpiration, and temperature. Agronomy Journal, 2009; 101(4): 854-864.

[3] Leteya J, Hoffmanb G J, Hopmansc J W, Grattan S R, Suarez D, Corwin D $\mathrm{L}$, et al. Evaluation of soil salinity leaching requirement guidelines. Agricultural Water Management, 2011; 98(4): 502-506.

[4] Liu S H, Kang Y H, Wan S Q, Wang Z C, Liang Z W, Sun X J. Water and salt regulation and its effects on Leymus chinensis growth under drip irrigation in saline-sodic soils of the Songnen Plain. Agricultural Water Management, 2011; 98(9): 1469-1476.

[5] Yuan S Q, Li H, Wang X K. Status, problems, trends and suggestions for water-saving irrigation equipment in China. Journal of Drainage and Irrigation Machinery Engineering, 2015; 33(1): 78-92.

[6] Lan C Y, Yi X T, Xue G N, Hou Y S. Chinese Academy of Agriculture Mechanization Sciences. Drainage and Irrigation Machinery, 2005; 23(1): $1-6$.

[7] Hao J D. The development and status of the hose reel irrigator in overseas market. Water Saving Irrigation, 1979; 3: 64-75.

[8] Xu Y F, Francis T E S T A. The characteristic analysis of the hose reel irrigator. Water Saving Irrigation, 1996; 3: 39-45.

[9] Tang Y, Zhu X Y, Mei X X, Gu Z. Performance test of water turbine of JP50 reel sprinkler. China Rural Water and Hydropower, 2014; 2: 26-29.

[10] Yuan S Q, Niu G P, Tang Y, Tang L D, Zhu X Y. Experiment and numerical estimation of performance of hydraulic turbine of JP50 reel sprinkle. Journal of Drainage and Irrigation Machinery Engineering, 2014; 37(7): 553-557.

[11] Niu G P. Hydraulic performance test and numerical simulation of water turbine of hose reel sprinkle. Jiangsu University, 2015. (in Chinese)

[12] Chen D X, Zhang W J, Yang L. Operation characteristics and type selection of cooling tower fan driven turbine. Water Power, 2010; 36(12): 54-56.

[13] Zhang L J, Wang L, Ren Y, Chen D X. Analysis on hydraulic characteristics of micro Francis hydro-turbine with low specific speed. Water Resources and Hydropower Engineering, 2013; 44(1): 128-130.

[14] Zhang L J, Chen D X, Ren Y, Li Y P. Hydraulic loss analysis of the micro Francis turbine of cooling towers. Journal of North China Institute of Water Conservancy and Hydroelectric Power, 2012; 33(1): 1-3. (in Chinese)

[15] Zhu F, Zheng Y, Fan X J, Yang C X, Li J. Design and numerical simulation of small Francis turbine used in cooling tower. Water Resources and Power, 2013; 31(7): 165-168.

[16] Zou K N, Li L, Xia K G. Hydraulic design and numerical simulation of super low specific speed Francis turbine runner. Energy Engineering, 2012; 4: 6-9.

[17] Jain S V, Patel R N. Investigations on pump running in turbine mode: A review of the state-of-the-art. Renewable and Sustainable Energy Reviews, 2014; 30: 841-868.

[18] Derakhshan S, Nourbakhsh A. Theoretical, numerical and experimental investigation of centrifugal pumps in reverse operation. Experimental Thermal and Fluid Science, 2008; 32: 1620-1627.

[19] Derakhshan S, Nourbakhsh A. Experimental study of characteristic curves of centrifugal pumps working as turbines in different specific speeds. Experimental Thermal and Fluid Science, 2008; 32:800-807.

[20] Shinhama H, Fukutomi J, Nakase Y, Chin Y, Kuwauchi T, Miyauchi S. Study on reverse running pump turbine. Transactions of the Japan Society of Mechanical Engineers (JSME), 1999; 65(638): 3399-3405. (in Japanese)

[21] González J, Oro J M F, Argüelles-Díaz K M, Santolaria C. Flow analysis for a double suction centrifugal machine in the pump and turbine operation modes. International Journal for Numerical Methods in Fluids, 2009; 61: 220-236.

[22] Fernández J, Barrio R, Blanco E, Parrondo J, Marcos A. Experimental and numerical investigation of a centrifugal pump working as a turbine. Proceedings of the ASME 2009 Fluids Engineering Division Summer Meeting FEDSM 2009, Vail, Colorado, USA, August 2-6, 2009; pp.1-9.

[23] Singh P, Nestmann F. Internal hydraulic analysis of impeller rounding in centrifugal pumps as turbines. Experimental Thermal and Fluid Science, 2011; 35(1): 121-134.

[24] Singh P. Optimization of the internal hydraulic and of system design in pumps as turbines with field implementation and evaluation. University of Karlsruhe, Karlsruhe, 2005.

[25] Derakhshan S, Mohammadi B. Incomplete sensitivities for 3D radial turbomachinery blade optimization. Computers \& Fluids, 2008; 37: 1354-1363.

[26] Derakhshan S, Mohammadi B, Nourbakhsh A. The comparison of incomplete sensitivities and Genetic algorithms applications in 3D radial turbomachinery blade optimization. Computer \& Fluids, 2010; 39: 2022-2029.

[27] Zhang M, Tsukamoto $H$. Unsteady hydrodynamic forces due to rotor-stator interaction on a diffuser pump with identical number of vanes on the impeller and diffuser. ASME Fluids Engineering, 2005; pp.743-751. 